\title{
Effect of Dietary Supplementation with L-Arginine and Vitamin C on Reproduction Performance and Economical Efficiency for Matrouh Strain Hens under Egyptian Summer Conditions
}

Elham S. Mohamed ${ }^{1 *}$, Abdella $^{2}$ M. M., El-Sayaad ${ }^{2}$ G. A. and Hassan ${ }^{1}$ I. I.

1- Animal Production Res. Inst., Agric. Res. Center, Dokki, Giza, Egypt

2- Poultry Production Dept., Fac. of Agric. Benha Univ., Egypt

*Corresponding author: elhamsalah985@gmail.com

\begin{abstract}
The present work was aimed to study the effect of dietary L-arginine level, vitamin C, and their interaction between them on some reproductive performance and economical efficiency of Matrouh laying hens during the summer season. A 4X3 factorial treatment arrangement was carried out including four levels of dietary L-Arg $(0.0,0.4,0.8$, and $1.2 \mathrm{~g} / \mathrm{kg}$ diet $)$ and three $\mathrm{VC}$ concentrations $(0,150$, and $300 \mathrm{mg} / \mathrm{kg} \mathrm{diet})$. A total number of 108 Matrouh hens and 36 cocks (Egyptian local strain) aged 64 weeks were randomly distributed into twelve treatment groups (three replicate each). The results obtained showed non-significant $(\mathrm{p}<0.05)$ effect in fertility percentages due to dietary L-Arg or VC supplementation through all the experimental periods. The interaction effects between dietary L-Arg and VC levels had a major influence $(\mathrm{p}<0.05)$ on fertility percentage. Dietary L-Arg levels had significant $(\mathrm{P}<0.05)$ effects on the hatchability percentages of total eggs set and fertile egg during all periods of estimation. The VC supplementation didn't have any significant effects on fertility and hatchability of fertile eggs percentage. The interaction effects between dietary L-Arg and VC levels had a major influence $(p<0.05)$ on the hatchability percentages of total eggs set during all the interval periods. In conclusion, it could be concluded that Matrouh laying hens supplemented diet with L-Arg at a level of $0.4 \mathrm{~g}$ and VC at a level of $150 \mathrm{mg} / \mathrm{kg}$ diet, seemed to be adequate to achieve the favorite results and is recommended.
\end{abstract}

Keywords: L-arginine, vitamin C, laying hens, fertility percentage, hatchability.

\section{Introduction}

The aims of the poultry industry are to increase the efficiency of the transformation from feed to animal proteins for consumers and to reduce the nitrogen excretion pollution. At present, synthetic amino acids are added to low-protein diets to obtain a wellbalanced feed. L-arginine (ARG), like most amino acids traditionally noted for their role in protein synthesis, is metabolized by arginases to L-ornithine for polyamine biosynthesis, and by nitric oxide synthases (NOS) to L-citrulline and nitric oxide (NO) (Yuan et al., 2016). However, there exist differences in the L-Arg requirement because most mature mammals are able to synthesize L-Arg to meet their requirements while uricotelic species like birds cannot synthesize L-Arg due to an incomplete urea cycle (D'Amato and Humphrey, 2010).

Arginine helps to avoid the harmful effects of excessive free radicals produced during body metabolism (Atakisi et al. 2009) as well as growth and reproduction (Fernandes et al. 2009). Arginine is essential for sperm motility, metabolism, capacitation, acrosome reaction, and is a precursor for producing putrescine, spermine, and spermidine (Ko and Sabangh, 2014). Spermidine is synthesized from putrescine and is a precursor of spermine. Daily LArg supplementation improves sperm concentration and motility (Scibona et al., 1994).

On the other hand, poultry are unable to synthesize ARG due to the lack of variant enzymes of the urea cycle (Fernandes et al., 2009).
Ascorbic acid is an antioxidant and water-soluble vitamin that is found intra- and extra cellular as ascorbate (Chihuailaf et al., 2002). In addition, it is a natural antioxidant that prevents the increased production of free radicals induced by oxidative damage to lipids and lipoproteins in various cellular compartments and tissues (Erdogan et al., 2005). Ascorbic acid supplementation at $200 \mathrm{ppm}$ is beneficial for enhancing the immunity, performance, and exploiting the broilers' full genetic potential (Lohakare et al., 2005).

Ascorbic acid can used as an anti-stress agent to minimize embryonic heat stress effects and have positive effects on embryos development and hatchling body weight under thermoneutral or heat stress conditions. (Kutlu, 2001, and Nowaczewski et al., 2012).

The role of vitamin $\mathrm{C}$ in the improvement of egg laying has not been sufficiently elucidated al- though various researchers indicate its involvement in the process of maturation of egg vesicles (Kolb and Seehawer, 2001). The beneficial effect on egg production of vitamin $\mathrm{C}$ added to feeds for laying hens was particularly evident when the birds were exposed to stress caused by high air temperature or increased stocking rate of birds per unit area (Njoku and Nwazota, 1989; Kontecka et al., 1997). Peebles and Brake (1985) were among those researchers who reported the influence of vitamin $\mathrm{C}$ added to the feed on the improvement of hatchability in broiler chickens. In addition, vitamin $\mathrm{C}$ was also reported to 
have a positive impact on the hatchability of Pekin ducks (Kontecka et al., 2001). Abdelfattah (2019) indicated that the rate of embryonic mortality was decreased remarkably due to treatment with ascorbic acid and thus increased hatching rate.

Therefore, the objective of the present study was planned to investigate the beneficial effects of dietary supplementation of L-arginine and ascorbic acid on some reproductive performances during the summer seasons for Matrouh chickens as local strain hens

\section{Materials and Methods}

The latest research was conducted at the Inshas Poultry Breeding Research Station, Animal Production Research Institute, Agriculture Research Center, Ministry of Agriculture, Giza, Egypt, during the period from May 2018 to August 2018. The chemical analysis was carried out at the Laboratories of the Animal Production Research Institute, Ministry of Agriculture. The experimental design of this work was planned to study the effect of adding different levels of L-arginine and vitamin C dietary supplementations on reproductive performances of Matrouh local strain from 64 to 75 weeks of layers age during the summer season of egypt. A total number of 108 laying hens and 36 cocks of Matrouh local strain, 64 weeks old, were randomly chosen from a large commercial flock. All selected hens and cocks were approximately of an equal body weight and similar performance. All birds of the experimental treatments were individually housed in wire-caged batteries $(45 \times 30 \times 35 \mathrm{~cm})$. All birds were reared under the same management and hygienic condition and fed a basal laying ration formulated according to the recommendation of NRC (1994). Feed ingredients and chemical analysis of the experimental basal diet are presented in Table (1). The photoperiod during the experimental period was fixed at $16 \mathrm{hrs}$ daily. Hens were fed ad-libitum and the fresh water was available all the time during the experimental period (12 weeks). All selected birds were randomly distributed into twelve groups ( 9 hens and 3 cocks / each). A $4 \mathrm{x}$ 3 factorial arrangement design was used in this experiment of design with four levels of L-arginine ( 0 , $0.4,0.8$ and $1.2 \mathrm{~g} / \mathrm{kg}$ diet) and three levels of Vitamin $\mathrm{C}(0,150$ and $300 \mathrm{mg} / \mathrm{kg}$ diet $)$ and their interaction were tested from 64 to 75 weeks of age. Cocks were used to collect semen and artificially inseminated to hens for two times per week. Experimental birds were divided into two groups. Birds of the first group fed either the basal diet only and conceded as control group or supplemented with $0,0.4,0.8$ or $1.2 \mathrm{~g} \mathrm{~L}$ arginine $/ \mathrm{kg}$ diet and the second group fed diet supplemented diet with Vitamin C (VC) at a level of 0,150 or $300 \mathrm{mg} / \mathrm{kg}$ diet, respectively. Small amounts of the basal diet were mixed at first with the respective amount of any supplement as a small batch, and then gradually mixed with a larger amount of the basal diet until the total amount of the respective diet was reached, finally the mixture was thoroughly homogenized mixed. At 68, 72 and 75 weeks of age, 30 eggs from each treatment group were chosen randomly at every four weeks and stored at $18^{\circ} \mathrm{C}$ temperature and $65 \%$ relative humidity. Eggs were incubated in an automatic computerized hatchery at the Poultry Breeding Station in Inshas. After hatching, the chicks were counted and non-hatched eggs were broken to determine the percentages of fertility and hatchability. Fertility was calculated as the percentage of the number of fertile eggs relative to the number of total eggs according to the following formula:

Fertility $\%=\frac{\text { Number of fertile eggs }}{\text { Number of all setting eggs }}$ X100

Hatchability was estimated as the percentage of the number of hatched chicks to the number of fertile eggs or the number of total eggs set as follows: -

Hatchability of total eggs set $\%=\frac{\text { Number of hatched eggs }}{\text { Number of total eggs set }}$ X100

Hatchability of fertile eggs $\%=\frac{\text { Number of hatched eggs }}{\text { Number of fertile eggs }} \times 100$

The economic efficiency (EE.) of egg production was calculated from the input and output analysis based upon the differences in egg production and feeding cost (Heady and Jensen, 1954). The REF were calculated as the net revenue per unit of total costs. Where:

$\mathrm{EE}=($ Net revenue/hen, LE / Total cost/hen, LE) $\mathrm{x} 100$.

*Price of $1 \mathrm{~kg} \mathrm{~L}-\mathrm{Arg}=320$ (LE)

* Price of $1 \mathrm{Kg} \mathrm{VC}=300$ (LE)

*Price of $1 \mathrm{Kg}$ gluten $=12.5(\mathrm{LE})$

$\mathrm{LE}=$ Egyptian pound. "According to the price prevailing at the local market at the experimental time. Data were statistically analyzed on factorial design (4x3) basis according to ANOVA procedures of SAS (SAS, 2012). Means differences were compared using Duncan's multiple range test (Duncan, 1955).

\section{Results and Discussion}

\section{Fertility percentage.}

Results obtained in Table (2) showed that dietary Larginine supplementation different levels had no significant effect on fertility percentages at all periods of estimation. Hens fed the $0.4 \mathrm{~g} / \mathrm{kg}$ diet L-arginine showed the higher average of fertility percentage, $(87.30 \%)$ when compared with different levels applied of L-arginine and control group. Results obtained agreed with those reported by Ezzat et al., (2015) affirmed that hatchability of fertile eggs were highest $(\mathrm{P} \leq 0.05)$ with $4.0 \mathrm{~g}$ L-arginine injection in air sac (90. 62\%) in Japanese quails. Also, Youssef et al., (2015) observed that significant increase in fertility and hatchability \% as compared with control group in local chicken. Dietary Vitamin C supplementation had 
no significant effect on average fertility percentages at all periods estimation. The higher average of fertility percentage $(86.50 \%)$ was attained by hens fed diet supplemented with $150 \mathrm{mg}$ V.C/kg diet. The interactions between dietary L-arginine and V.C supplementation were significant $(\mathrm{P}<0.05)$ on average fertility percentages during the interval periods, except at the period from $72--75$ weeks of age. The higher averages of fertility percentage $(90.48 \%)$ was recorded in the hens feed diet supplemented with $1.2 \mathrm{~g}$ L-arginine $/ \mathrm{kg}$ diet without V.C supplementation (table,2). While, the lower ones $(76.18 \%)$ was observed in the hens recorded diet $1.2 \mathrm{~g} \mathrm{~L}$-arginine $/ \mathrm{kg}$ diet and 300mg V.C $/ \mathrm{kg}$ diet when compared with different treatment.

\section{Hatchability percentage of total eggs set.}

Results obtained in Table (3) revealed that dietary L-arginine supplementation of different level had significant $(\mathrm{P}<0.05)$ effect on hatchability percentages of total eggs set during all the experimental periods. The higher hatchability percentage of total eggs set $(35.97 \%)$ was recorded in hens fed diet supplementation with $1.2 \mathrm{~g}$ L-arginine $/ \mathrm{kg}$ diet. This is due to 1-arginine stimulates release of gastrointestinal hormones encouraging cell division, protein synthesis and tissue growth. And also increased villi heights in duodenum and ileum of birds resulting from in ovo injection of L-arginine. Dietary V.C supplementation had significant $(\mathrm{P}<0.05)$ effects on hatchability percentages of total eggs set at all the experimental periods studied, except at the period from (64-67) wks of age. The higher hatchability percentage $(35.71 \%)$ of total eggs set was shown by hens fed diet supplemented with $150 \mathrm{mg}$ V.C $/ \mathrm{kg}$ diet. The interactions between dietary L-arginine and V.C supplementation had significant $(\mathrm{P}<0.05)$ on hatchability percentage of total eggs set at all the interval periods The higher averages of hatchability percentage $(50.79 \%)$ of total eggs set were recorded in hens fed diet supplementation with $1.2 \mathrm{~g} \mathrm{~L}$-arginine without V.C supplementation, While, the lowest one $(20.63 \%)$ was observed in the hens received diet supplementation $0.8 \mathrm{~g} \mathrm{~L}$ - arginine without V.C supplementation, when compared with different treatment.

\section{Hatchability percentage of fertile eggs.}

Data presented in Table (4) showed the averages of hatchability percentage of fertile eggs different groups of layers at all periods of estimation. Results obtained showed significant $(\mathrm{P}<0.05)$ effect of dietary Larginine levels on hatchability percentage of fertile eggs at all period. If could be noticed that percentages of hatchability were increased with increasing dietary
L-arginine level. Hens fed diet with $1.2 \mathrm{~g} \mathrm{~L}$-arginine level had record the higher average $(42.02 \%)$ of hatchability percentage of fertile egg. No significant effect of dietary V.C supplementation was observed on hatchability percentages of fertile eggs during all the experimental periods studied, except during the period from 72-75 weeks of hens age which showed significant $(\mathrm{P}<0.05)$ effect on hatchability percentages of fertile eggs. Hens fed diet supplemented with $150 \mathrm{mg}$ V.C $/ \mathrm{kg}$ recoded the higher average of hatchability percentage $(41.32 \%)$. Similar results were reported by Abdelfattah (2019) who declared that treatment with ascorbic acid increased hatching rate compared with the untreated group. Dietary Larginine and V.C supplementation interactions had significant $(\mathrm{P}<0.05)$ effect on hatchability of fertile eggs percentages at all periods of the experiment. The higher hatchability percentages $(55.69 \%)$ were observed in the interaction between $1.2 \mathrm{~g}$ dietary Larginine $/ \mathrm{kg}$ diet without V.C supplementation. Whereas, the lowest percentage $(26.59 \%)$ was observed in the interaction between $0.8 \mathrm{~g}$ dietary Larginine level without V.C supplementation.

\section{Economical efficiency.}

Results obtained revealed that the $0.4 \mathrm{~g}$ dietary Larginine level recorded the best relative economical efficiency (EEf) being $440 \%$ and diet supplemented with $150 \mathrm{mg} \mathrm{V} . \mathrm{C} / \mathrm{kg}$ diet attained the higher relative (EEf) value, being $133 \%$. When compared with different treatments applied and control group (Table $5)$. The higher average of EEf $(680 \%)$ was found in the interaction between $1.2 \mathrm{~g}$ dietary L-arginine level with 300mg V.C $/ \mathrm{kg}$ diet, whereas, the lower one (20\%) was shown due to dietary supplementation with $300 \mathrm{mg}$ V.C $/ \mathrm{kg}$ diet without L-arginine supplementation. These results were agreed with those stated by Ezzat et al., (2015) who demonstrated that the highest net revenue chicks and best economic efficiency (EEF) values were recorded for fertile eggs injected with $4 \% \mathrm{~L}$-arginine in air sac followed by those $1 \% \mathrm{~L}$-arginine followed by $2 \% \mathrm{~L}$-arginine at 1 days of incubation when compared with other treatments.

\section{Conclusion}

In conclusion, the results of this study indicate that feeding Matrouh laying hens on diets containing $0.4 \mathrm{~g}$ L-arginine or $150 \mathrm{mg}$ V.C $/ \mathrm{kg}$ diet $/ \mathrm{kg}$ diet and the interaction between dietary $1.2 \mathrm{~g}$ L-arginine with $300 \mathrm{mg}$ V.C $/ \mathrm{kg}$ diet improved the reproductive performance and economical efficiency of Matrouh laying hens. 
Table 1. Feed ingredients and chemical analysis of the basal laying diet.

\begin{tabular}{ll}
\hline Ingredients & $\%$ \\
\hline Yellow corn $(8.5 \%)$ & 62.80 \\
Wheat Bran $(15.7 \%)$ & 10.00 \\
Soybean meal $(44 \%)$ & 9.40 \\
& 8.00 \\
Limestone $\left(\mathrm{CaCo}_{3}\right)$ & 6.70 \\
Di-calcium phosphate & 3.32 \\
Salt (NaCl) & 0.34 \\
Dl-Methionine 99\% & 0.03 \\
L-Lysine HCL & 0.11 \\
Vit + Min. premix* & 0.30 \\
& 100 \\
Chemical analysis: - & \\
a-Calculated analysis $* *:-$ & \\
ME, Kcal/kg. & 2728 \\
Crude protein, \% & 16.00 \\
Crude fiber, \% & 3.14 \\
EE & 3.05 \\
Calcium, \% & 3.33 \\
Ash \% & 3.14 \\
Available phosphorus, \%. & 0.73 \\
Lysine, \%. & 0.53 \\
Methionine, \% & 0.72 \\
Arginine & 0.75 \\
b- Determined analysis $* * *:-$ & \\
Crude protein, \%. & 16.00 \\
Crude fiber, \%. & 3.14 \\
Ash \%. & 3.14 \\
\hline Vit Min. prem: Eal & \\
\hline
\end{tabular}

*Vit. Min. premix: Each $2 \mathrm{~kg}$ of vitamin and mineral premix (Commercial source AGRIVET Co.) contains Vit. A. 12000000 IU, Vit. D3 2000000 IU, Vit. E. 10000 mg, Vit. K3 2000 mg, Vit. B1 100 mg, Vit. B2 5000 mg, Vit. B6 1500 mg, Vit. B12 $10 \mathrm{mg}$, Biotin $50 \mathrm{mg}$, Choline chloride $250000 \mathrm{mg}$, Pantothenic acid $10000 \mathrm{mg}$, Nicotinic acid $3000 \mathrm{mg}$, Folic acid $1000 \mathrm{mg}$, Manganese $60000 \mathrm{mg}$, Zinc $50000 \mathrm{mg}$, Iron $30000 \mathrm{mg}$, Copper $10000 \mathrm{mg}$, Iodine $1000 \mathrm{mg}$, Selenium $100 \mathrm{mg}$, Cobalt $100 \mathrm{mg}$, Carrier $\left(\mathrm{Ca} \mathrm{CO}_{3}\right)$ add to $2 \mathrm{~kg}$.

** Calculated in accordance with NRC (1994).

*** Determined according to the styles of AOAC (2005).

Table 2. Least square means and standard error $(\bar{X} \pm S E)$ for fertility percentage as affected by dietary supplementation.

\begin{tabular}{|c|c|c|c|c|c|}
\hline \multirow[b]{2}{*}{ Items } & & \multicolumn{4}{|c|}{ Fertility percentage (\%) at wks } \\
\hline & & 64-67 & 68-71 & $72-75$ & $\begin{array}{c}\text { Average } \\
(64-75)\end{array}$ \\
\hline \multicolumn{2}{|c|}{ L-arginine levels (g/kg diet) } & NS & NS & NS & NS \\
\hline \multicolumn{2}{|c|}{0.0} & $85.71 \pm 3.78$ & $82.53 \pm 2.71$ & $83.36 \pm 4.33$ & $83.87 \pm 2.58$ \\
\hline \multicolumn{2}{|c|}{0.4} & $88.88 \pm 2.53$ & $87.30 \pm 2.52$ & $85.71 \pm 4.60$ & $87.30 \pm 2.35$ \\
\hline \multicolumn{2}{|c|}{0.8} & $82.54 \pm 2.06$ & $76.19 \pm 2.30$ & $88.88 \pm 2.91$ & $82.54 \pm 1.64$ \\
\hline \multicolumn{2}{|c|}{1.2} & $85.71 \pm 3.99$ & $80.95 \pm 3.75$ & $85.71 \pm 3.33$ & $84.12 \pm 3.18$ \\
\hline \multicolumn{2}{|c|}{ Vitamin C levels (mg/kg diet) } & NS & NS & NS & NS \\
\hline \multicolumn{2}{|c|}{0} & $85.71 \pm 3.22$ & $83.33 \pm 2.57$ & $82.76 \pm 4.12$ & $83.93 \pm 2.25$ \\
\hline \multicolumn{2}{|c|}{150} & $88.09 \pm 2.30$ & $83.33 \pm 2.16$ & $88.09 \pm 3.09$ & $86.50 \pm 1.78$ \\
\hline \multicolumn{2}{|c|}{300} & $83.33 \pm 2.64$ & $78.57 \pm 3.11$ & $86.90 \pm 2.34$ & $82.93 \pm 2.36$ \\
\hline \multicolumn{6}{|c|}{ Interaction effects: } \\
\hline \multirow[t]{2}{*}{$\begin{array}{c}\text { L-arginine levels } \\
\text { (g/kg diet) }\end{array}$} & $\begin{array}{c}\text { Vitamin C levels } \\
\text { (mg/kg diet) }\end{array}$ & $*$ & $*$ & NS & $*$ \\
\hline & 0 & $71.43 \pm 2.75^{\mathrm{d}}$ & $85.71 \pm 2.85^{\mathrm{ab}}$ & $73.91 \pm 0.79$ & $77.01 \pm 1.93^{\mathrm{ab}}$ \\
\hline \multirow[t]{3}{*}{0.0} & 150 & $95.23 \pm 0.64^{\mathrm{a}}$ & $85.71 \pm 3.89^{\mathrm{ab}}$ & $85.71 \pm 12.10$ & $88.89 \pm 5.25^{\mathrm{ab}}$ \\
\hline & 300 & $90.47 \pm 2.37^{\mathrm{ab}}$ & $76.19 \pm 5.92^{\mathrm{ab}}$ & $90.47 \pm 2.34$ & $85.71 \pm 3.28^{\mathrm{ab}}$ \\
\hline & 0 & $95.23 \pm 1.56^{\mathrm{a}}$ & $85.71 \pm 4.61^{\mathrm{ab}}$ & $80.95 \pm 14.43$ & $87.30 \pm 6.27^{\mathrm{ab}}$ \\
\hline \multirow[t]{2}{*}{0.4} & 150 & $85.71 \pm 5.81^{\mathrm{abc}}$ & $90.47 \pm 3.75^{\mathrm{a}}$ & $90.47 \pm 2.76$ & $88.89 \pm 2.81^{\mathrm{ab}}$ \\
\hline & 300 & $85.71 \pm 3.28^{\mathrm{abc}}$ & $85.71 \pm 5.76^{\mathrm{ab}}$ & $85.71 \pm 3.92$ & $85.71 \pm 4.08^{\mathrm{ab}}$ \\
\hline
\end{tabular}




\begin{tabular}{ccllll}
\hline & 0 & $80.95 \pm 3.66^{\mathrm{bcd}}$ & $71.43 \pm 3.07^{\mathrm{b}}$ & $90.47 \pm 3.62$ & $80.95 \pm 0.62^{\mathrm{ab}}$ \\
0.8 & 150 & $85.71 \pm 4.65^{\mathrm{abc}}$ & $76.19 \pm 3.98^{\mathrm{ab}}$ & $85.71 \pm 6.68$ & $82.54 \pm 4.10^{\mathrm{ab}}$ \\
& 300 & $80.95 \pm 2.92^{\mathrm{bcd}}$ & $80.95 \pm 3.95^{\mathrm{ab}}$ & $90.47 \pm 6.05$ & $84.13 \pm 3.55^{\mathrm{ab}}$ \\
& 0 & $95.23 \pm 1.07^{\mathrm{a}}$ & $90.47 \pm 2.20^{\mathrm{a}}$ & $85.71 \pm 8.79$ & $90.48 \pm 3.61^{\mathrm{a}}$ \\
1.2 & 150 & $85.71 \pm 5.21^{\mathrm{abc}}$ & $80.95 \pm 1.05^{\mathrm{ab}}$ & $90.47 \pm 1.00$ & $85.71 \pm 2.04^{\mathrm{ab}}$ \\
& 300 & $76.19 \pm 8.48^{\mathrm{cd}}$ & $71.43 \pm 8.52^{\mathrm{b}}$ & $80.95 \pm 5.71$ & $76.18 \pm 7.16^{\mathrm{b}}$ \\
\hline
\end{tabular}

$\overline{(\bar{X} \pm S E)}=$ Average \pm standard error

a,b. and c means having different letters at the same column are significantly $(\mathrm{P} \leq 0.05)$ different.

$*=(\mathrm{P} \leq 0.05) ; \mathrm{NS}=$ Not significant

Table 3. Least square means and standard error $(\bar{X} \pm S E)$ for hatchability $(\%)$ of total eggs set as affected by dietary supplementation.

\begin{tabular}{|c|c|c|c|c|c|}
\hline \multirow{2}{*}{\multicolumn{2}{|c|}{ Items }} & \multicolumn{4}{|c|}{ Hatchability percentage of total eggs set $(\%)$ at wks } \\
\hline & & 64-67 & 68-71 & 72-75 & $\begin{array}{c}\text { Average } \\
(64-75)\end{array}$ \\
\hline \multicolumn{2}{|c|}{ L-arginine levels (g/kg diet) } & $*$ & $*$ & $*$ & $*$ \\
\hline \multicolumn{2}{|c|}{0.0} & $47.61 \pm 3.95^{\mathrm{b}}$ & $34.92 \pm 2.90^{\mathrm{ab}}$ & $18.77 \pm 3.34^{\mathrm{a}}$ & $33.77 \pm 2.42^{\mathrm{ab}}$ \\
\hline \multicolumn{2}{|c|}{0.4} & $47.61 \pm 3.15^{\mathrm{b}}$ & $38.09 \pm 3.01^{\mathrm{a}}$ & $9.52 \pm 1.58^{\mathrm{b}}$ & $31.74 \pm 1.33^{\mathrm{b}}$ \\
\hline \multicolumn{2}{|c|}{0.8} & $41.27 \pm 3.83^{\mathrm{b}}$ & $34.92 \pm 3.41^{\mathrm{ab}}$ & $23.81 \pm 5.24^{\mathrm{a}}$ & $33.33 \pm 3.50^{\mathrm{ab}}$ \\
\hline \multicolumn{2}{|c|}{1.2} & $55.55 \pm 3.34^{\mathrm{a}}$ & $30.15 \pm 5.14^{\mathrm{b}}$ & $22.22 \pm 5.124^{\mathrm{a}}$ & $35.97 \pm 4.2^{\mathrm{a}}$ \\
\hline \multicolumn{2}{|c|}{ Vitamin C levels (mg/kg diet) } & NS & $*$ & $*$ & $*$ \\
\hline \multicolumn{2}{|c|}{0} & $48.80 \pm 4.05$ & $36.90 \pm 3.62^{\mathrm{a}}$ & $15.27 \pm 4.25^{\mathrm{b}}$ & $33.66 \pm 3.48^{\mathrm{ab}}$ \\
\hline \multicolumn{2}{|c|}{150} & $47.61 \pm 3.21$ & $35.71 \pm 2.12^{\mathrm{ab}}$ & $23.81 \pm 2.92^{\mathrm{a}}$ & $35.71 \pm 1.69^{\mathrm{a}}$ \\
\hline \multicolumn{2}{|c|}{300} & $47.61 \pm 2.87$ & $30.95 \pm 3.61^{b}$ & $16.66 \pm 3.83^{\mathrm{b}}$ & $31.74 \pm 2.32^{\mathrm{b}}$ \\
\hline \multicolumn{6}{|c|}{ Interaction effects: } \\
\hline \multirow[t]{2}{*}{$\begin{array}{c}\text { L-arginine } \\
\text { levels (g/kg } \\
\text { diet) }\end{array}$} & $\begin{array}{l}\text { Vitamin C levels } \\
\text { (mg/kg diet) }\end{array}$ & $*$ & $*$ & $*$ & $*$ \\
\hline & 0 & $42.85 \pm 4.74^{\text {cde }}$ & $28.57 \pm 1.82^{\mathrm{cd}}$ & $8.69 \pm 2.73^{\mathrm{d}}$ & $26.71 \pm 1.85^{\text {fgh }}$ \\
\hline \multirow[t]{2}{*}{0.0} & 150 & $61.90 \pm 2.01^{\mathrm{ab}}$ & $42.85 \pm 4.84^{\mathrm{ab}}$ & $19.04 \pm 1.71^{\mathrm{bc}}$ & $41.27 \pm 1.59^{\mathrm{b}}$ \\
\hline & 300 & $38.09 \pm 1.59^{\mathrm{de}}$ & $33.33 \pm 4.64^{\mathrm{bcd}}$ & $28.57 \pm 4.993^{\mathrm{ab}}$ & $33.33 \pm 3.33^{\text {cde }}$ \\
\hline \multirow{3}{*}{0.4} & 0 & $52.38 \pm 0.23^{\mathrm{bc}}$ & $47.62 \pm 4.31^{\mathrm{a}}$ & $9.52 \pm 2.64^{\mathrm{d}}$ & $36.50 \pm 1.35^{\mathrm{bcd}}$ \\
\hline & 150 & $38.09 \pm 2.82^{\mathrm{de}}$ & $33.33 \pm 0.73^{\mathrm{bcd}}$ & $14.28 \pm 0.33^{\mathrm{cd}}$ & $28.57 \pm 0.87^{\mathrm{efg}}$ \\
\hline & 300 & $52.38 \pm 6.61^{b c}$ & $33.33 \pm 4.66^{\mathrm{bcd}}$ & $4.76 \pm 0.43$ & $30.16 \pm 1.01^{\mathrm{def}}$ \\
\hline \multirow{3}{*}{0.8} & 0 & $33.33 \pm 3.06^{\mathrm{e}}$ & $23.81 \pm 2.45^{\mathrm{de}}$ & $4.76 \pm 1.56^{\mathrm{d}}$ & $20.63 \pm 0.55^{\mathrm{h}}$ \\
\hline & 150 & $38.09 \pm 2.75^{\mathrm{de}}$ & $38.09 \pm 3.72^{\mathrm{abc}}$ & $38.09 \pm 4.28^{\mathrm{a}}$ & $38.09 \pm 3.57^{b c}$ \\
\hline & 300 & $52.38 \pm 7.81^{\mathrm{bc}}$ & $42.85 \pm 4.69^{\mathrm{ab}}$ & $28.57 \pm 3.77^{\mathrm{ab}}$ & $41.26 \pm 3.32^{\mathrm{b}}$ \\
\hline \multirow{3}{*}{1.2} & 0 & $66.66 \pm 5.17^{\mathrm{a}}$ & $47.62 \pm 5.14^{\mathrm{a}}$ & $38.09 \pm 5.28^{\mathrm{a}}$ & $50.79 \pm 1.35^{\mathrm{a}}$ \\
\hline & 150 & $52.38 \pm 1.83^{\mathrm{bc}}$ & $28.57 \pm 2.08^{\mathrm{cd}}$ & $23.81 \pm 2.73^{b}$ & $34.92 \pm 1.71^{\text {bcde }}$ \\
\hline & 300 & $47.62 \pm 2.44^{\mathrm{cd}}$ & $14.28 \pm 2.69^{\mathrm{e}}$ & $4.76 \pm 0.36^{\mathrm{d}}$ & $22.22 \pm 1.48^{\mathrm{gh}}$ \\
\hline
\end{tabular}

$\overline{(\bar{X} \pm S E)}=$ Average \pm standard error

a,b.c,d and e means having different letters at the same column are significantly $(\mathrm{P} \leq 0.05)$ different.

$*=(\mathrm{P} \leq 0.05) ; \mathrm{NS}=$ Not significant 
Table 4. Least square means and standard error $(\bar{X} \pm S E)$ for hatchability $(\%)$ of fertile eggs as affected by dietary supplementation.

\begin{tabular}{|c|c|c|c|c|c|}
\hline \multirow{2}{*}{\multicolumn{2}{|c|}{ Items }} & \multicolumn{4}{|c|}{ Hatchability of fertile eggs (\%) at wks } \\
\hline & & 64-67 & 68-71 & 72-75 & $\begin{array}{c}\text { Average } \\
(64-75)\end{array}$ \\
\hline \multicolumn{2}{|c|}{ L-arginine levels (g/kg diet) } & $*$ & $*$ & $*$ & $*$ \\
\hline \multicolumn{2}{|c|}{0.0} & $55.70 \pm 4.03^{\mathrm{b}}$ & $42.36 \pm 2.87^{\mathrm{b}}$ & $21.85 \pm 3.67^{\mathrm{a}}$ & $39.97 \pm 1.69^{a}$ \\
\hline \multicolumn{2}{|c|}{0.4} & $53.51 \pm 3.08^{b}$ & $43.76 \pm 3.41^{\mathrm{a}}$ & $11.03 \pm 1.71^{b}$ & $36.10 \pm 1.62^{\mathrm{b}}$ \\
\hline \multicolumn{2}{|c|}{0.8} & $50.10 \pm 3.90^{\mathrm{b}}$ & $45.42 \pm 3.94^{\mathrm{a}}$ & $27.09 \pm 6.03^{\mathrm{a}}$ & $40.87 \pm 3.83^{\mathrm{a}}$ \\
\hline \multicolumn{2}{|c|}{1.2} & $64.53 \pm 2.41^{\mathrm{a}}$ & $35.97 \pm 4.91^{b}$ & $25.54 \pm 5.92^{\mathrm{a}}$ & $42.02 \pm 3.88^{\mathrm{a}}$ \\
\hline \multicolumn{2}{|c|}{ Vitamin C levels (mg/kg diet) } & NS & NS & $*$ & NS \\
\hline \multicolumn{2}{|c|}{0} & $56.54 \pm 3.41$ & $43.71 \pm 3.47$ & $18.30 \pm 4.81^{\mathrm{b}}$ & $39.52 \pm 3.28$ \\
\hline \multicolumn{2}{|c|}{150} & $53.74 \pm 3.32$ & $43.03 \pm 2.79$ & $27.19 \pm 3.59^{a}$ & $41.32 \pm 1.89$ \\
\hline \multicolumn{2}{|c|}{300} & $57.60 \pm 3.13$ & $38.89 \pm 3.86$ & $18.65 \pm 4.24^{\mathrm{b}}$ & $38.38 \pm 2.41$ \\
\hline \multicolumn{6}{|c|}{ Interaction effects: } \\
\hline \multirow[t]{2}{*}{$\begin{array}{l}\text { L-arginine levels } \\
\text { (g/kg diet) }\end{array}$} & $\begin{array}{c}\text { Vitamin C levels } \\
\text { (mg/kg diet) }\end{array}$ & $*$ & $*$ & $*$ & $*$ \\
\hline & 0 & $60.00 \pm 2.62^{\mathrm{ab}}$ & $33.33 \pm 3.78^{c}$ & $11.76 \pm 1.72^{\mathrm{de}}$ & $35.03 \pm 1.17^{\mathrm{de}}$ \\
\hline \multirow[t]{3}{*}{0.0} & 150 & $65.00 \pm 6.34^{\mathrm{ab}}$ & $50.00 \pm 1.84^{\mathrm{ab}}$ & $22.22 \pm 5.49^{\mathrm{bcd}}$ & $45.74 \pm 1.13^{\mathrm{bc}}$ \\
\hline & 300 & $42.10 \pm 1.83^{\mathrm{d}}$ & $43.75 \pm 3.23^{\mathrm{abc}}$ & $31.58 \pm 5.49^{b}$ & $39.14 \pm 1.64^{\mathrm{d}}$ \\
\hline & 0 & $55.00 \pm 3.43^{\mathrm{bc}}$ & $55.55 \pm 3.86^{\mathrm{a}}$ & $11.76 \pm 1.86^{\mathrm{de}}$ & $40.77 \pm 2.97^{\mathrm{cc}}$ \\
\hline \multirow[t]{3}{*}{0.4} & 150 & $44.44 \pm 4.80^{\mathrm{cd}}$ & $36.84 \pm 4.13^{c}$ & $15.79 \pm 2.16^{\mathrm{cde}}$ & $32.36 \pm 1.60^{\mathrm{ef}}$ \\
\hline & 300 & $61.11 \pm 2.87^{\mathrm{ab}}$ & $38.89 \pm 1.66^{\mathrm{bc}}$ & $5.55 \pm 0.71^{\mathrm{e}}$ & $35.18 \pm 1.32^{\mathrm{de}}$ \\
\hline & 0 & $41.17 \pm 2.17^{\mathrm{d}}$ & $33.33 \pm 1.53^{c}$ & $5.26 \pm 0.58^{e}$ & $26.59 \pm 0.85^{f}$ \\
\hline \multirow[t]{2}{*}{0.8} & 150 & $44.44 \pm 0.40^{\mathrm{cd}}$ & $50.00 \pm 6.77^{\mathrm{ab}}$ & $44.44 \pm 2.93^{\mathrm{a}}$ & $46.30 \pm 3.16^{b c}$ \\
\hline & 300 & $64.70 \pm 3.89^{\mathrm{ab}}$ & $52.94 \pm 5.16^{\mathrm{a}}$ & $31.58 \pm 5.44^{\mathrm{b}}$ & $49.74 \pm 3.15^{b}$ \\
\hline \multirow{3}{*}{1.2} & 0 & $70.00 \pm 4.22^{\mathrm{a}}$ & $52.63 \pm 3.98^{a}$ & $44.44 \pm 5.77^{\mathrm{a}}$ & $55.69 \pm 0.97^{\mathrm{a}}$ \\
\hline & 150 & $61.11 \pm 1.73^{\mathrm{ab}}$ & $35.29 \pm 2.73^{\mathrm{c}}$ & $26.31 \pm 3.73^{b c}$ & $40.91 \pm 1.75^{\mathrm{cc}}$ \\
\hline & 300 & $62.50 \pm 5.14^{\mathrm{ab}}$ & $20.00 \pm 0.52^{\mathrm{d}}$ & $5.88 \pm 1.08^{\mathrm{e}}$ & $29.46 \pm 1.96^{\mathrm{ef}}$ \\
\hline
\end{tabular}

$(\bar{X} \pm S E)=$ Average \pm standard error

a,b.c,de and f means having different letters at the same column are significantly $(\mathrm{P} \leq 0.05)$ different.

$*=(\mathrm{P} \leq 0.05) ; \mathrm{NS}=$ Not significant

Table 5. Economical efficiency of egg production of Matrouh layers as affected by dietary L-arginine levels, Vitamin $\mathrm{C}$ and them interactions at the end of the experimental period (at 75 weeks of age).

\begin{tabular}{|c|c|c|c|c|c|c|c|c|c|c|c|c|}
\hline \multicolumn{2}{|c|}{ Items } & $\begin{array}{c}\text { Egg } \\
\text { number } \\
\text { (hen/period) }\end{array}$ & $\begin{array}{l}\text { Price/ } \\
\text { egg } \\
\text { (LE) }\end{array}$ & $\begin{array}{c}\text { Total } \\
\text { revenue } \\
\text { hen } \\
(\text { LE) }\end{array}$ & $\begin{array}{c}\text { Total } \\
\text { feed } \\
\text { intake/ } \\
\text { hen }(\mathbf{k g})\end{array}$ & $\begin{array}{c}\text { Price } \\
/ \text { Kg } \\
\text { feed } \\
\text { (LE) }\end{array}$ & $\begin{array}{c}\text { Total } \\
\text { feed } \\
\text { cost/ } \\
\text { hen } \\
\text { (LE) }\end{array}$ & $\begin{array}{l}\text { Fixed } \\
\text { / hen } \\
\text { price } \\
\text { (LE) }\end{array}$ & $\begin{array}{l}\text { Total } \\
\text { cost } \\
\text { hen } \\
(\mathrm{LE})\end{array}$ & $\begin{array}{c}\text { Net } \\
\text { revenue/ } \\
\text { hen } \\
\text { (LE) }\end{array}$ & $\begin{array}{l}\text { Economical } \\
\text { efficiency } \\
\text { (EEf) }\end{array}$ & $\begin{array}{r}\text { Relative } \\
\text { EEf \% }\end{array}$ \\
\hline \multicolumn{13}{|c|}{$\begin{array}{l}\text { L-arginine levels } \\
\text { (g/kg diet) }\end{array}$} \\
\hline 0. & & 33.64 & 1.00 & 33.64 & 7.84 & 3.763 & 29.50 & 2.40 & 31.90 & 1.74 & 0.05 & 100 \\
\hline 0. & & 38.16 & 1.00 & 38.16 & 7.39 & 3.891 & 28.75 & 2.40 & 31.15 & 7.01 & 0.22 & 440 \\
\hline 0. & & 35.71 & 1.00 & 35.71 & 6.83 & 4.019 & 27.44 & 2.40 & 29.84 & 5.87 & 0.19 & 380 \\
\hline 1. & & 37.75 & 1.00 & 37.75 & 6.99 & 4.147 & 28.98 & 2.40 & 31.38 & 6.37 & 0.20 & 400 \\
\hline \multicolumn{13}{|c|}{$\begin{array}{l}\text { Vitamin C levels } \\
\text { (mg /kg diet) }\end{array}$} \\
\hline 0 & & 34.98 & 1.00 & 34.98 & 7.22 & 3.763 & 27.16 & 2.40 & 29.56 & 5.42 & 0.18 & 100 \\
\hline 15 & & 36.46 & 1.00 & 36.46 & 7.14 & 3.763 & 26.86 & 2.40 & 29.26 & 7.20 & 0.24 & 133 \\
\hline 30 & & 37.50 & 1.00 & 37.50 & 7.43 & 3.763 & 27.95 & 2.40 & 30.35 & 7.15 & 0.23 & 128 \\
\hline $\begin{array}{c}\text { Interactior } \\
\text { L- } \\
\text { arginine } \\
\text { levels } \\
\text { (g/kg } \\
\text { diet) }\end{array}$ & $\begin{array}{c}n \text { effect } \\
\text { Vitami } \\
\text { C level } \\
(\mathrm{mg} / \mathrm{k} \\
\text { diet) }\end{array}$ & & & & & & & & & & & \\
\hline 0.0 & $\begin{array}{c}0 \\
150 \\
\end{array}$ & $\begin{array}{l}31.57 \\
35.34 \\
\end{array}$ & $\begin{array}{l}1.00 \\
1.00 \\
\end{array}$ & $\begin{array}{l}31.57 \\
35.34 \\
\end{array}$ & $\begin{array}{l}7.35 \\
7.82 \\
\end{array}$ & $\begin{array}{l}3.763 \\
3.763 \\
\end{array}$ & $\begin{array}{l}27.65 \\
29.42 \\
\end{array}$ & $\begin{array}{l}2.40 \\
2.40 \\
\end{array}$ & $\begin{array}{l}30.05 \\
31.82 \\
\end{array}$ & $\begin{array}{l}1.52 \\
3.52 \\
\end{array}$ & $\begin{array}{l}0.05 \\
0.11 \\
\end{array}$ & $\begin{array}{l}100 \\
220 \\
\end{array}$ \\
\hline
\end{tabular}




\begin{tabular}{ccccccccccccc}
\hline & 300 & 34.01 & 1.00 & 34.01 & 8.35 & 3.763 & 31.42 & 2.40 & 33.82 & 0.19 & 0.01 & 20 \\
& 0 & 39.31 & 1.00 & 39.31 & 7.52 & 3.891 & 29.26 & 2.40 & 31.66 & 7.65 & 0.24 & 480 \\
0.4 & 150 & 37.17 & 1.00 & 37.17 & 6.89 & 3.891 & 26.80 & 2.40 & 29.20 & 7.97 & 0.27 & 540 \\
& 300 & 37.99 & 1.00 & 37.99 & 7.77 & 3.891 & 30.23 & 2.40 & 32.63 & 5.36 & 0.16 & 320 \\
& 0 & 32.59 & 1.00 & 32.59 & 6.78 & 4.019 & 27.24 & 2.40 & 29.64 & 2.95 & 0.09 & 180 \\
0.8 & 150 & 37.27 & 1.00 & 37.27 & 6.86 & 4.019 & 27.57 & 2.40 & 29.97 & 7.30 & 0.24 & 480 \\
& 300 & 37.27 & 1.00 & 37.27 & 6.84 & 4.019 & 27.48 & 2.40 & 29.88 & 7.39 & 0.24 & 480 \\
& 0 & 36.45 & 1.00 & 36.45 & 7.24 & 4.147 & 30.02 & 2.40 & 32.42 & 4.03 & 0.12 & 240 \\
1.2 & 150 & 36.05 & 1.00 & 36.05 & 6.99 & 4.147 & 28.98 & 2.40 & 31.38 & 4.67 & 0.14 & 280 \\
& 300 & 40.74 & 1.00 & 40.74 & 6.74 & 4.147 & 27.95 & 2.40 & 30.35 & 10.39 & 0.34 & 680 \\
\hline
\end{tabular}

Total revenue $=$ Egg number $/$ hen X Price/egg (LE).

Net revenue/hen $(\mathrm{LE})=$ Total revenue - Total cost $/$ hen .
Fixed hen (LE) $=$ Rearing cost.

EEf $=$ Net revenue/hen $(\mathrm{LE}) /$ Total cost/hen $(\mathrm{LE})$.

\section{References}

A.O.A.C. (2005): Official Methods of Analysis. $18^{\text {th }}$ Ed. Association of Official Analytical Chemists, Gaithersburg, USA.

Abdelfattah, M.G. (2019). Effect of thermal manipulation during incubation and spraying Japanese quail eggs with ascorbic acid on embryogenesis and phsiological responses of hatch chicks. Egypt. Poult. Sci. Vol. (39):479-499.

Atakisi, O.; Atakisi, E. and A. Kart (2009). Effects of dietary zinc and L-arginine supplementation on total antioxidants capacity, lipid peroxidation, nitric oxide, egg weight and blood biochemical values in Japanese quails. Biological Trace Element Research 132: 136 - 143.

Chihuailaf, R.; Contreras, P. and F. Wittwer (2002). Pathogenesis of oxidative stress: Consequences and evaluation in animal health. Vet. México, 33: 265-283.

D'Amato, J. L., and B. D. Humphrey. (2010). Dietary arginine levels alter markers of arginine utilization in peripheral blood mononuclear cells and thymocytes in young broiler chicks. Poult. Sci. 89:938-947.

Duncan, D. B. (1955). The Multiple Range and Multiple F-Tests. Biometrics, 11: 1-42.

Erdogan, Z.; Erdogan, S.; Celik, S. and A. Unlu, (2005). Effects of ascorbic acid on cadmiuminduced oxidative stress and performance of broilers. Biol. Trace Elem. Res., 104:19-31.

Ezzat, W.; A. A. Habib and A. A. El- Zaiat (2015). The effect of 1-arginine injection by different doses and sites in fertile eggs on hatchability, productive performance and some physiological parameters of Japanese quail's post- hatching. J. Product. \& Dev., 20 (3): 395 - 411.

Fernandes, J.I.; Murakami, A.E; Martins, E.N.; Sakamoto, M.I. and E.R. Garcia (2009). Effect of arginine on the development of the pectoralis muscle and the diameter and the protein: Deoxyribonucleic acid rate of its skeletal myofibers in broilers. Poultry Science 88: 1399 1406.
Heady, E.O. and H. R. Jensen (1954). Farm Management Economics. Prentic- Hall ,Inc. Englewood Cliffs, N.J.

Ko, E. Y. and E. S. Sabanegh (2014). The role of nutraceuticals in male fertility. Urologic Clinics of North America, 41: 181-193.

Kolb E. and J. Seehawer (2001). Bedeutung und Anwendung der Ascorbinsäure beim Geflügel. Arch. Geflügelkd. 65, 106-113.

Kontecka H., Książkiewicz J., Nowaczewski S., Kisiel T., and S. Krystianiak (2001). Effect of feed supplementation with vitamin $\mathrm{C}$ on reproduction results in Pekin ducks. In: Proc. $9^{\text {th }}$ Int. Symp. Current Problems of Breeding, Health and Production of Poultry, 6-7 luty. České Budějovice, Czech Republic, 42. (sited by Czech J. Anim. Sci., 50, 2005 (5): 208-212)

Kontecka K., Witkiewicz K., and J. Sobczak (1997). Wpływ dodatku witaminy $C$ do paszy na wskaźniki produkcyjne i hematologiczne kur nieśnych. Przegl. Hod. Zesz. Nauk. PTZ, 32, 139145. (sited by Czech J. Anim. Sci., 50, 2005 (5): 208-212)

Kutlu, H.R. (2001). Influences of wet feeding and supplementation with ascorbic acid on performance and carcass composition of broiler chicks exposed to a high ambient temperature. Arch. Anim. Nutri., 54: 127- 139.

Lohakare, J.D.; Kim, J.K.; Ryu, M.H.; Hahn, T.W. and B.J. Chae (2005). Effects of vitamin $C$ and vitamin $\mathrm{D}$ interaction on the performance, immunity, and bone characteristics of commercial broilers. J. Appl. Poult. Res., 14:670-678.

Njoku P.C., and A.O.U. Nwazota (1989). Effect of dietary inclusion of ascorbic acid and palm oil on the performance of laying hens in hot tropical environment. Brit. Poultry Sci., 30, 831-840. (sited by Czech J. Anim. Sci., 50, 2005 (5): 208212)

Nowaczewski, S.; Kontecka, H., and S. Krystianiak., (2012). Effect of in ovo injection of vitamin $\mathrm{C}$ during incubation on hatchability of chickens and ducks. Folia Biol. 60: 93-97. 
NRC (1994). National Research Council, Nutrient Requirements of Poultry. $9^{\text {th }}$ Ed, National Academic of Science. Washington, DC. USA.

Peebles E. D., and J. Brake (1985). Relationship of dietary ascorbic acid to broiler breeder performance. Poultry Sci., 64, 2041-2048. (sited by Czech J. Anim. Sci., 50, 2005 (5): 208-212)

SAS (2012). SAS for Windows. Version 9.4. SAS Institute Inc. Cary, NC, USA.

Scibona, M.; Meschini, P.; Capparelli, S.; Pecori, C.; Rossi, P. and F. G. Menchini (1994). Larginine and male infertility. Minerva urologica e nefrologica. 46: 251-253.
Youssef, S. F.; S. A. M. Shaban and Inas, I. Ismail (2015). Effect of L- arginine supplementation on productive, reproductive performance, immune response and gene expression in tow local chicken strains. 1-Egg production, reproductive performance and immune response. Egypt. Poult. Sci., $35: 573590$.

Yuan, C.; X. C. Bu; H. X. Yan; J. J. Lu and X. T. Zou (2016). Dietary L-arginine levels affect the liver protein turnover and alter the expression of genes related to protein synthesis and proteolysis of laying hens. Poult. Sci. 95:261-267.

$$
\begin{aligned}
& \text { تأثير التغذية بإضافة الأرجنين وفيتامين C للعليقة على الكفاءة التناسلية والاقتصادية لدجاج سلالة مطروح. }
\end{aligned}
$$

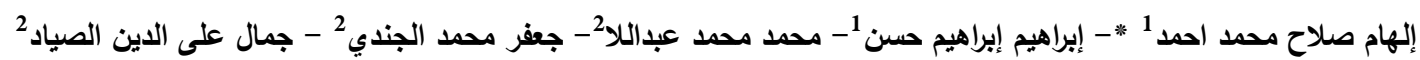

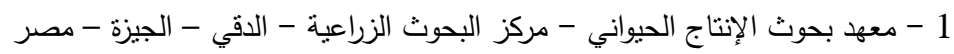

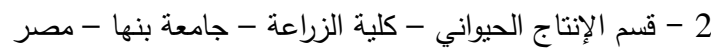

*Corresponding author: elhamsalah985@gmail.com

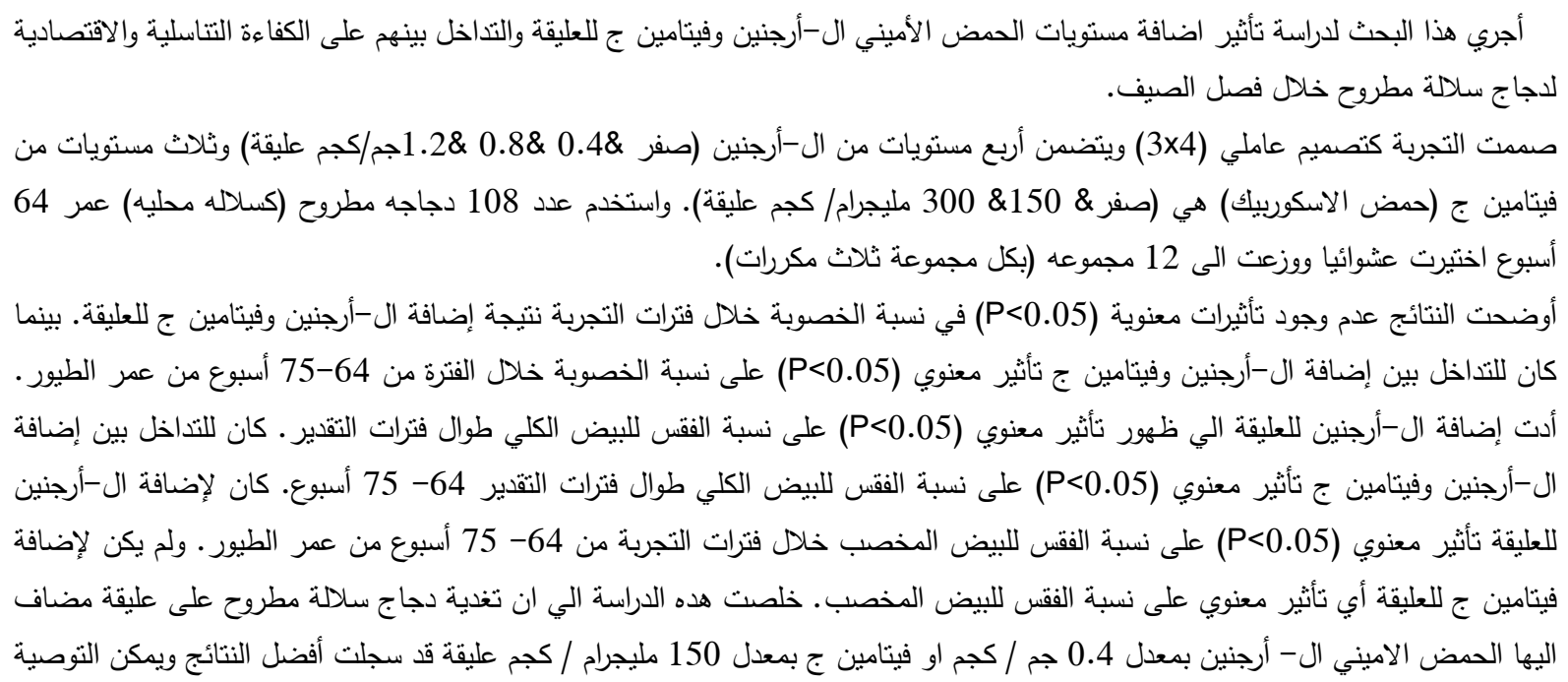

